\title{
Cométabolisme sucre-acides aminés chez Lactococcus lactis
}

\author{
Levente Novak, Muriel Cocaign-Bousquet, Nicholas D. Lindley, \\ Pascal Loubière
}

\begin{abstract}
Centre de bio-ingénierie Gilbert-Durand, UMR CNRS 5504 et laboratoire associé Inra, Institut national des sciences appliquées, Complexe scientifique de Rangueil, 31077 Toulouse cedex 4, France
\end{abstract}

\begin{abstract}
Cometabolism sugar-amino acids in Lactococcus lactis. The growth of Lactococcus lactis subsp. lactis NCDO 2118 was directly related to the medium composition as regards both the growth yield and the growth rate. The growth yield relative to the catabolic substrate, glucose, and the maximun specific growth rate increased with the medium complexity. Growth was limited by the cells' capacity to feed the anabolic pathways, while the catabolic rate was always proportional to the anabolic rate thereby avoiding energy spoilage. The conversion yield of glucose to fermentation products was constant whatever the medium composition. Approximately $88 \%$ of the glucose was recovered as classical fermentation products and, in a minimal medium containing 6 amino acids, a further $5 \%$ in biomass accounting for $60 \%$ of the total biomass. Inversely, some of the amino acids were converted to fermentation products. () Inra/Elsevier, Paris.
\end{abstract}

\section{Lactococcus lactis / amino acid / growth / metabolism / energetics}

\begin{abstract}
Résumé - La croissance de Lactococcus lactis subsp. lactis NCDO 2118 est dépendante de la composition du milieu de culture, tant en terme d'efficacité que de vitesse. Le rendement en biomasse formée par rapport au substrat catabolique, le glucose, augmente avec la richesse du milieu, de même que le taux de croissance. La croissance est limitée par les capacités de l'anabolisme, alors que le catabolisme s'adapte à l'anabolisme de façon à éviter un gaspillage d'énergie. Si la vitesse du catabolisme est d'autant plus rapide que l'anabolisme est efficace, le rendement de conversion du sucre en produits de fermentation reste constant quel que soit le milieu de culture. Sur un milieu minimal contenant six acides aminés, le glucose participe pour $88 \%$ à la synthèse de produits de fermentation, et pour $5 \%$ à la synthèse de biomasse, correspondant à $60 \%$ de la biomasse formée. Inversement, les acides aminés sont à l'origine de certains produits minoritaires de fermentation. (c) Inra/Elsevier, Paris.
\end{abstract}

Lactococcus lactis / acide aminé / croissance / métabolisme / énergétique 


\section{INTRODUCTION}

Les bactéries lactiques ont une importance considérable dans le domaine agroalimentaire. Pourtant, leur croissance reste, aujourd'hui encore, un phénomène mal contrôlé et fastidieux, qui nécessite un apport complexe en éléments nutritifs. Si les milieux de culture complexes à base de peptone, extrait de levure ou tryptone, couramment utilisés, permettent d'assurer une bonne croissance, les phénomènes métaboliques et énergétiques mis en jeu ne peuvent être étudiés dans ces conditions. L'étude du rôle des acides aminés en relation avec la biosynthèse cellulaire est ainsi complètement négligé. Pourtant, il est clairement démontré que la croissance peut être améliorée grâce à l'addition d'extrait de levure [9], de peptone [3] ou d'acides aminés [6] dans le milieu de culture.

Il est couramment admis, selon une schématisation simpliste, que catabolisme et anabolisme sont quasi totalement dissociés du point de vue des flux carbonés, et reliés simplement par le flux énergétique. Ce schéma repose sur le fait que le glucose est effectivement transformé en grande majorité en produits de fermentation. De plus, Schmitt et al. [8] ont montré que le glucose n'était pas impliqué dans l'anabolisme de Leuconostoc mesenteroides, mais que la croissance était réalisée sur un milieu complexe, en présence de glucose et de citrate, alors que ce dernier avait une fonction anabolique, À l'heure actuelle, aucune étude complète n'a été réalisée pour étudier l'origine de la biomasse dans un milieu synthétique. Outre l'étude du devenir du glucose dans la biomasse, un deuxième intérêt réside dans l'étude de la répartition des acides aminés, dont certains peuvent avoir une fonction catabolique intéressante pour la production de molécules à caractère aromatique, dans le réseau métabolique.
Divers milieux synthétiques définis, construits grâce à une étude complète des besoins nutritionnels de Lactococcus lactis NCDO 2118 [2], sont utilisés pour la croissance de cette souche. Les substrats, glucose et acides aminés, les produits de fermentation et la biomasse sont mesurés en vue de comparer, en terme de bilan et de vitesse, le comportement de la souche visà-vis du carbone, de l'azote et de l'énergie. Les substrats (glucose et acides aminés) marqués au ${ }^{14} \mathrm{C}$ sont utilisés pour caractériser le devenir de chacun d'eux dans la totalité du réseau métabolique, non seulement vers les produits de fermentation, mais aussi dans les différentes macromolécules constituant la biomasse.

\section{MATÉRIEL ET MÉTHODES}

La souche utilisée dans cette étude est Lactococcus lactis subsp. lactis NCDO 2118. Elle est conservée à $-80^{\circ} \mathrm{C}$ dans du milieu chimiquement défini (MCD) décrit par Poolman et Konings [6], supplémenté de glycérol (20\%). Cinq milieux sont utilisés pour la croissance : le milieu complexe M17 [10], le milieu MCD contenant 47 éléments dont 18 acides aminés et 14 vitamines, les milieux synthétiques simplifiés mis au point par Cocaign-Bousquet et al. [2], MS 10 contenant 27 éléments dont les mêmes 18 acides aminés que le MCD, MS13 contenant 15 éléments dont 6 acides aminés, et enfin MS14 différent du MS13 dans la concentration des acides aminés [4]. Les cultures sont réalisées à $30^{\circ} \mathrm{C}, \mathrm{pH} 6,6$, sous atmosphère d'azote, et agitées à $250 \mathrm{rpm}$. Les conditions de culture et les méthodes d'analyse du glucose, des acides aminés, de la biomasse, de l'ammoniaque et des produits de fermentation sont identiques à celles précédemment décrites [4].

L'analyse de la biomasse (protéines, lipides, acides nucléiques...) est réalisée selon les méthodes classiques d'analyse. Le marquage de la biomasse est effectué en incubant les cellules dans le milieu MS14 additionné de 9 à $60 \mathrm{kBq} / \mathrm{mL}$ de glucose ou des différents acides aminés du MS14, glutamate, sérine, valine, isoleucine ou leucine, uniformément marqués au ${ }^{14} \mathrm{C}$. La biomasse marquée est fractionnée selon la méthode de Paalme et al. [5], et chaque fraction comptée dans un compteur à scin- 
tillation (Beckman LS 1801) après addition de $4 \mathrm{~mL}$ de cocktail de scintillation par $\mathrm{mL}$ de fraction. La radioactivité dans les produits de fermentation est mesurée en couplant l'analyse en HPLC avec un compteur à scintillation en continu (Berthold LB 506A).

\section{RÉSULTATS ET DISCUSSION}

\subsection{Croissance de $L$. lactis sur différents milieux}

Grâce à la mesure précise de la consommation du glucose et des acides aminés, de la formation de la biomasse, éventuellement d'ammoniaque, et des produits du métabolisme, les fermentations sont analysées en terme de bilan global sur le carbone, l'azote et l'énergie, ou en terme de vitesses de réactions. Quel que soit le milieu de culture utilisé, la réaction catabolique de conversion du glucose en produits classiques de fermentation est équilibrée à $88 \%$; sur la base de 100 $\mathrm{mmol} / \mathrm{L}$ de glucose consommés, c'est-àdire $600 \mathrm{mmol} / \mathrm{L}$ de carbone, $528 \mathrm{mmol} / \mathrm{L}$ de carbone sont retrouvés dans ces produits, dont 510 en lactate. Il est clair qu'une partie du glucose est donc déviée, soit vers des produits de fermentation non identifiés, soit vers la biomasse. Le bilan de production d'ATP est, lui aussi, quasi constant sur tous les milieux, avec un rendement de 1,8 mol ATP/mol glucose.

Le bilan carboné de la réaction anabolique de synthèse de la biomasse à partir des acides aminés est identique sur MCD et MS10, milieux de composition identique en acides aminés : $100 \mathrm{C}$-aminoacides donnent $94 \mathrm{C}$-biomasse. Ce bilan est plus amplement déficitaire sur MS13 où $75 \mathrm{C}$-aminoacides donnent $57 \mathrm{C}$-biomasse, soit un taux de recouvrement de $76 \%$. Une partie des acides aminés consommés donne donc, au moins sur milieu minimum, des produits de fermentation. Une conclusion identique découle du bilan azoté, puisque le taux de recou- vrement de l'azote est inférieur à $100 \%$, avec des valeurs de $70 \%$ sur MCD et MS10 et de $80 \%$ sur MS13, et dans ce dernier cas, $16 \mathrm{~N}$-aminoacides sont convertis en $11 \mathrm{~N}$-biomasse plus $2 \mathrm{NH}_{3}$. Certains des produits de fermentation issus des acides aminés sont donc des produits azotés non identifiés. Sur chacun de ces milieux, étant donné que les acides aminés sont en partie à l'origine de produits de fermentation, une partie du glucose doit donc avoir une fonction anabolique.

Le rendement global apparent montre que la richesse nutritionnelle du milieu détermine l'efficacité de la croissance. En effet, le rendement en biomasse évolue de $14 \mathrm{~g}$ biomasse $/ \mathrm{mol}$ glucose sur MS13 à $33 \mathrm{~g} / \mathrm{mol}$ sur M17 (tableau I). Le rendement identique observé sur MCD et MS10 montre qu'il est déterminé par la composition en acides aminés et indépendant de la présence de bases nucléotidiques, oligoéléments ou vitamines. Parallèlement, les valeurs de $Y_{\text {ATP }}$ augmentent de $8 \mathrm{~g}$ biomasse/mol ATP sur MS13, à $15 \mathrm{~g} / \mathrm{mol}$ sur MCD et MS10, et $17 \mathrm{~g} / \mathrm{mol}$ sur M17, valeurs cohérentes avec celles calculées par Benthin et al. [1] pour Lactococcus cremoris. La variabilité observée pour les valeurs de $\mathrm{Y}_{\text {ATP }}$ montre que la croissance n'est pas limitée par l'énergie, mais par l'anabolisme.

Les vitesses spécifiques maximales de croissance augmentent, elles aussi, avec la richesse du milieu de culture (tableau I). La richesse nutritionnelle du milieu détermine donc la vitesse de croissance. De plus, si en terme de rendement de croissance, les milieux MCD et MS10 étaient équivalents, la présence supplémentaire de bases nucléotidiques permet d'accélérer la croissance, comme précédemment observé [2]. Alors que l'on aurait pu s'attendre à ce que la vitesse spécifique de consommation du glucose soit maintenue constante sur tous les milieux, comme décrit pour la croissance de Streptococcus bovis sur différents milieux [7], 
Tableau I. Paramètres énergétiques et de croissance observés lors de la culture de L. lactis NCDO 2118 sur différents milieux.

Table I. Energetic and growth parameters observed during growth of L. lactis NCDO 2118 in various media.

\begin{tabular}{lcccc}
\hline Milieu & $\begin{array}{c}\mathrm{Y}_{\mathrm{X}}{ }^{1} \\
(\mathrm{~g} / \mathrm{mol})\end{array}$ & $\begin{array}{c}\mathrm{Y}_{\mathrm{ATP}}{ }^{2} \\
(\mathrm{~g} / \mathrm{mol})\end{array}$ & $\begin{array}{c}\mu_{\max } \\
\left(\mathrm{h}^{-1}\right)\end{array}$ & $\begin{array}{c}\mathrm{q}_{\mathrm{s}}{ }^{\max } \\
(\mathrm{mmol} / \mathrm{g} . \mathrm{h})\end{array}$ \\
\hline M17 & 33,0 & 17,6 & 1,10 & 25,0 \\
MCD & 28,5 & 16,0 & 0,80 & 24,5 \\
MS10 & 26,2 & 15,0 & 0,60 & 20,6 \\
MS13 & 14,6 & 8,0 & 0,23 & 15,7 \\
\hline
\end{tabular}

${ }^{1} \mathrm{Y}_{\mathrm{X}}$ : rendement en biomasse en $\mathrm{g}$ biomasse par mol de substrat. ${ }^{2} \mathrm{Y}_{\mathrm{ATP}}$ : rendement ATP en $g$ biomasse par mol d'ATP.

${ }^{1} \mathrm{Y}_{\mathrm{X}}$ : growth yield in $g$ biomass per mol substrate. ${ }^{2} \mathrm{Y}_{\text {ATP }}$ : ATP yield in $g$ biomass per ATP.

elle varie avec la composition du milieu entre $15,7 \mathrm{mmol} . \mathrm{g}^{-1} \cdot \mathrm{h}^{-1}$ sur MS13 et $25 \mathrm{mmol} \cdot \mathrm{g}^{-1} \cdot \mathrm{h}^{-1}$ sur M17. Étant donné que le flux anabolique est limitant et dépendant de la richesse du milieu, le flux catabolique s'adapte au flux anabolique pour éviter une perte d'énergie.

\subsection{Répartition des substrats dans le réseau métabolique}

La croissance de L. lactis en présence de substrats uniformément marqués au ${ }^{14} \mathrm{C}$ permet de caractériser le devenir du carbone dans le réseau métabolique. Le milieu MS14 contenant seulement six acides aminés est un excellent modèle pour ce type d'études. La répartition de chacun des substrats présents dans le milieu, c'est-à-dire glucose, sérine, glutamate, isoleucine, leucine et valine (hormis la méthionine très faiblement consommée), dans le réseau métabolique est ainsi étudiée par la mesure du ${ }^{14} \mathrm{C}$ dans l'ensemble des molécules issues du catabolisme ou de l'anabolisme.

L'analyse en HPLC des produits de fermentation montre que, outre les produits classiques de la fermentation homolactique, d'autres produits minoritaires sont détectés. Le ${ }^{14} \mathrm{C}$-glucose marque logiquement les produits directement issus du métabolisme central via le pyruvate, c'està-dire le lactate, le formiate et l'acétate, mais ne marque aucun des autres produits. Le même résultat est obtenu à partir de la sérine qui, dans ce milieu contenant peu d'acides aminés, est en grande partie désaminée en pyruvate [4]. Les autres produits de fermentation excrétés en faible concentration dans le milieu de culture sont marqués à partir des quatre autres acides aminés testés. La radioactivité issue du glutamate se retrouve dans quatre de ces produits minoritaires, dont le pyroglutamate et l' $\alpha$-cétoglutarate. La valine marque un sous-produit important (pic à $21 \mathrm{~min}$ ), tandis que 1'isoleucine et la leucine marquent le produit minoritaire dont la réponse en HPLC est la plus élevée (pic à $28 \mathrm{~min}$ ). Il est ainsi clairement établi que les acides aminés ont une fonction catabolique et qu'ils sont à l'origine de plusieurs produits de fermentation.

L'analyse de la composition macromoléculaire de la biomasse permet d'établir le pourcentage de chaque fraction par rapport à la biomasse totale, les protéines représentant la fraction la plus importante (tableau $I$ ). Le fractionnement de la biomasse après croissance en présence du 
Tableau II. Composition macromoléculaire de L. lactis cultivée sur MS14, obtenue par fractionnement séquentiel de la biomasse (exprimée en pourcentage de la biomasse) et répartition du glucose dans ces différentes fractions (exprimée en pourcentage de la fraction marquée par le ${ }^{14} \mathrm{C}$-glucose).

Table II. Macromolecular composition of L. lactis cultivated in MS14 medium obtained by sequential fractionation of biomass (expressed in percentage of the total biomass), and glucose distribution in the different fractions (expressed in percentage of the fraction labelled by the ${ }^{14} \mathrm{C}$-glucose).

\begin{tabular}{lcc}
\hline Fraction & Pourcentage de la biomasse & $\begin{array}{c}\text { Pourcentage de marquage } \\
\text { à partir du glucose }\end{array}$ \\
\hline Pools & 5 & 48 \\
Lipides & 4 & 81 \\
ARN & 6 & 85 \\
ADN et sucres & 28,5 & 71 \\
Protéines et aminosucres & 50,5 & 44 \\
\hline
\end{tabular}

glucose ou de chacun des acides aminés marqués au ${ }^{14} \mathrm{C}$, suivi de la mesure du marquage dans chaque fraction, montre que le glucose marque toutes les fractions macromoléculaires à un taux variant de 44 à $85 \%$ (tableau II). La sérine, étant donné qu'elle est désaminée en pyruvate, apparaît également de façon minoritaire dans chaque fraction. C'est logiquement dans les protéines que la part du glucose est la plus faible ( $44 \%$ ), et où les acides aminés participent en majorité. L'hydrolyse des protéines montre que le glucose marque en totalité l'histidine et les acides aminés aromatiques, dérivés des intermédiaires glycolytiques en amont du phosphoénolpyruvate. Il participe aussi pour $70 \%$ à la synthèse de la glycine, les $30 \%$ restants étant issus de la sérine. Les acides aminés synthétisés en aval du pyruvate (alanine et famille de l'aspartate) proviennent à $90 \%$ du glucose et à $10 \%$ de la sérine. Le glutamate est à l'origine des acides aminés de sa propre famille. Enfin, les acides aminés branchés, présents dans le milieu de culture, ne sont à l'origine d'aucun autre aminoacide en quantité significative. Le carbone de la biomasse formée dans ce milieu synthétique provient donc à $60 \%$ du glucose, $15 \%$ du glutamate, $10 \%$ de la sérine, et $5 \%$ de la valine, de la leucine et de l'isoleucine. La part du glucose à l'origine de la biomasse correspond à $5 \%$ du glucose consommé, valeur certes faible par rapport à la totalité du glucose consommé, mais amplement représentative par rapport à la biomasse formée.

\section{RÉFÉRENCES}

[1] Benthin S., Schulze U., Nielsen J., Villadsen J., Growth energetics of Lactococcus cremoris FD1 during energy-, carbon-, and nitrogenlimitation in steady state and transient cultures, Chem. Engin. Sci. 49 (1994) 589-609.

[2] Cocaign-Bousquet M., Garrigues C., Novak L., Lindley N.D., Loubière P., Rational development of a simple synthetic medium for the sustained growth of Lactococcus lactis, J. Appl. Bacteriol. 79 (1995) 108-116.

[3] Law B.A., Sezgin E., Sharpe M.E., Amino acid nutrition of some commercial cheese starters in relation to their growth in peptonesupplemented whey media, J. Dairy Res. 43 (1976) 291-300.

[4] Novak L., Cocaign-Bousquet M., Lindley N.D., Loubière P., Metabolism and energetics of Lactococcus lactis during growth in various complex or synthetic media, Appl. Environ. Microbiol. 63 (1997) 2665-2670.

[5] Paalme T., Olivson A, Vilu R., ${ }^{13} \mathrm{C}-\mathrm{NMR}$ study of $\mathrm{CO}_{2}$-fixation during the heterotrophic growth in Chlorobium thiosulfatophilum, Biochim. Biophys. Acta 720 (1982) 311-319. 
[6] Poolman B., Konings W.N., Relation of growth of Streptococcus cremoris to amino acid transport, J. Bacteriol. 170 (1988) 700-707.

[7] Russell J.B., Effect of amino acids on the heat production and growth efficiency of Streptococcus bovis: balance of anabolic and catabolic rates, Appl. Environ. Microbiol. 59 (1993) 1747-1751.

[8] Schmitt P., Divies C., Cardona R., Origin of end-products from the co-metabolism of glu- cose and citrate by Leuconostoc mesenteroides subsp. cremoris, Appl. Microbiol. Biotechnol. 36 (1992) 679-683.

[9] Smith J.S., Hillier A.J., Lees G.J., The nature of the stimulation of the growth of Streptococcus lactis by yeast extract, J. Dairy Res. 42 (1975) 123-138.

[10] Terzaghi B., Sandine W.E., Improved medium for lactic streptococci and their bacteriophages, Appl. Microbiol. 29 (1975) 807-813. 